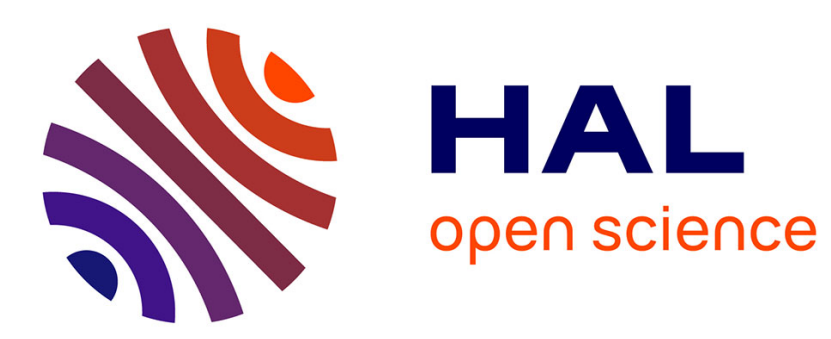

\title{
Cellules solaires CdS/CuInS2, préparées par pulvérisation chimique sans air
}

N. Kamoun, S. Belgacem, M. Dachraoui, R. Bennaceur

\section{To cite this version:}

N. Kamoun, S. Belgacem, M. Dachraoui, R. Bennaceur. Cellules solaires CdS/CuInS2, préparées par pulvérisation chimique sans air. Revue de Physique Appliquée, 1987, 22 (9), pp.991-998. 10.1051/rphysap:01987002209099100 . jpa-00245659

\section{HAL Id: jpa-00245659 https://hal.science/jpa-00245659}

Submitted on 1 Jan 1987

HAL is a multi-disciplinary open access archive for the deposit and dissemination of scientific research documents, whether they are published or not. The documents may come from teaching and research institutions in France or abroad, or from public or private research centers.
L'archive ouverte pluridisciplinaire HAL, est destinée au dépôt et à la diffusion de documents scientifiques de niveau recherche, publiés ou non, émanant des établissements d'enseignement et de recherche français ou étrangers, des laboratoires publics ou privés. 


\title{
Cellules solaires $\mathrm{CdS} / \mathrm{CuInS}_{2}$, préparées par pulvérisation chimique sans air
}

\author{
N. Kamoun, S. Belgacem, M. Dachraoui (*) et R. Bennaceur \\ Laboratoire de Physique de la Matière condensée, Faculté des Sciences de Tunis, Campus Universitaire, 1060 \\ Tunis, Tunisie \\ (*) Laboratoire de Chimie Analytique, Faculté des Sciences de Tunis, Campus Universitaire, 1060 Tunis, \\ Tunisie
}

(Reçu le 13 mars 1987, accepté le 18 mai 1987)

\begin{abstract}
Résumé. - Nous avons étudié les propriétés structurales et optiques des couches minces de $\mathrm{CuInS}_{2}$ préparées par pulvérisation chimique réactive sans air. L'analyse par diffraction aux rayons $\mathbf{X}$ a montré que ces couches sont bien cristallisées et leur orientation principale (112) est nettement privilégiée pour un rapport de concentrations $\frac{(\mathrm{Cu}(\mathrm{I}))}{(\operatorname{In}(\mathrm{III}))}=1$ dans la solution à pulvériser. La valeur de la largeur de bande interdite, de l'ordre de $1,45 \mathrm{eV}$, a été obtenue à partir des mesures de transmission et de réflexion optiques. Les valeurs de la tension de circuit ouvert et de courant de court-circuit, de la photopile $\mathrm{CdS} / \mathrm{CuInS}_{2}$ réalisée, sont respectivement de l'ordre de $0,3 \mathrm{~V}$ et $2,7 \mathrm{~mA} . \mathrm{cm}^{-2}$.
\end{abstract}

\begin{abstract}
We have studied the structural and optical properties of $\mathrm{CuInS}_{2}$ thin layers prepared by airless spray. The X-ray diffraction analysis showed that these layers are well crystallized with (112) principal orientation which is clearly privileged for a concentration ratio $\frac{(\mathrm{Cu}(\mathrm{I}))}{(\mathrm{In}(\mathrm{III}))}=1$ in the pulverization solution. The value of the gap, of the order of $1.45 \mathrm{eV}$, was obtained from transmission and reflection optical measurements. The values of open circuit voltage and short circuit current of $\mathrm{CdS} / \mathrm{CuInS} \mathrm{S}_{2}$ cell are respectively of the order of $0.3 \mathrm{~V}$ and $2,7 \mathrm{~mA} . \mathrm{cm}^{-2}$.
\end{abstract}

\section{Introduction.}

$\mathrm{Au}$ cours de ces dernières années la fabrication des composés ternaires I-III-VI $\mathrm{I}_{2}$ tels que $\mathrm{CuInX}_{2}(\mathrm{X}=\mathrm{S}$, $\mathrm{Se}, \mathrm{Te})$, utilisés en couches minces en tant qu'absorbeurs dans un dispositif photovoltaïque, a connu une attention considérable [1].

Le semiconducteur $\mathrm{CuInS}_{2}$ que nous avons étudié dans le cadre de ce travail présente en effet de bonnes caractéristiques photovoltaïques :

i) un coefficient d'absorption important $(\alpha \simeq$ $5 \times 10^{2} \mathrm{~cm}^{-1}-10^{5} \mathrm{~cm}^{-1}$ [2] ;

ii) un gap direct de valeur $E_{g} \simeq 1,42 \mathrm{eV}-$ $1,53 \mathrm{eV}$ voisin de la valeur théorique optimale pour la conversion de l'énergie solaire $(1,5 \mathrm{eV})$ [2-5] ; et

iii) il peut être fabriqué à caractère $\mathrm{p}$ ou $\mathrm{n}$ (par contrôle de l'excès ou de la déficience des atomes de soufre) permettant ainsi la réalisation d'homojonctions $[6,7]$ ou d'hétérojonctions telle que CdS/ $\mathrm{CuInS}_{2}[3,6,8]$.
Dans ce travail, nous avons étudié les propriétés structurales et optiques des couches minces de $\mathrm{CuInS}_{2}$ préparées par pulvérisation chimique réactive sans air [9-11] et nous avons testé les performances photovoltaïques des cellules solaires utilisant ces couches.

\section{Conditions expérimentales.}

Contrairement à la méthode de pulvérisation pneumatique, proposée pour la première fois par Chamberlin et Skarman [12], la pulvérisation réactive sans air se fait sans gaz vecteur à l'aide d'un pistolet à peinture (Wagner, type 320) [10, 11]. La formation du jet de liquide se fait à travers un orifice calibré $(\varnothing=0,5 \mathrm{~mm})$ d'un cylindre de compression sur des substrats en verre ordinaire de $3 \mathrm{~mm}$ d'épaisseur et chauffés à une température de $340^{\circ} \mathrm{C}$ $\left( \pm 5^{\circ} \mathrm{C}\right)$. Le débit de pulvérisation utilisé est de l'ordre de $25 \mathrm{ml} . \mathrm{min}^{-1}$. Dans ces conditions, pour des températures plus faibles, les couches minces de 
$\mathrm{CuInS}_{2}$ deviennent très inhomogènes et très rugueuses présentant ainsi une mauvaise cristallinité ; de même, à des températures plus hautes, l'épaisseur des couches est très faible. La composition de la solution utilisée pour la préparation des couches minces de $\mathrm{CuInS}_{2}$ est :

- Chlorure d'indium $\mathrm{InCl}_{3}$ :

$$
3,1 \times 10^{-2} \mathrm{~mol} .1^{-1}-6 \times 10^{-2} \mathrm{~mol} .1^{-1}
$$

- Chlorure cuivreux $\mathrm{CuCl}$ :

$$
3,1 \times 10^{-2} \mathrm{~mol} .1^{-1}-6 \times 10^{-2} \mathrm{~mol} . \mathrm{l}^{-1}
$$

- Thiourée $\mathrm{SC}\left(\mathrm{NH}_{2}\right)_{2}$ :

$$
0,15 \mathrm{~mol} . \mathrm{1}^{-1}-0,25 \mathrm{~mol} .1
$$

- Chlorure d'ammonium $\mathrm{NH}_{4} \mathrm{Cl}$ :

$$
1,25 \mathrm{~mol} . \mathrm{l}^{-1}
$$

- Dichlorohydrate d'hydrazine $\mathrm{N}_{2} \mathrm{H}_{4}, 2 \mathrm{HCl}$ :

$$
2,9 \times 10^{-3} \mathrm{~mol} \cdot \mathrm{1}^{-1}-0,56 \mathrm{~mol} \cdot \mathrm{1}^{-1}
$$

La réaction globale de formation des couches sur les substrats est :

$$
\begin{aligned}
\mathrm{CuCl}+\mathrm{InCl}_{3}+2 \mathrm{SC}\left(\mathrm{NH}_{2}\right)_{2}+ \\
+4 \mathrm{H}_{2} \mathrm{O} \rightarrow \mathrm{CuInS}_{2}+2 \mathrm{CO}_{2}+4 \mathrm{NH}_{4} \mathrm{Cl} .
\end{aligned}
$$

Les couches étudiées ont une épaisseur variant de $0,3 \mu \mathrm{m}$ à $1 \mu \mathrm{m}$.

L'étude de la cristallinité et de l'orientation des dépôts de $\mathrm{CuInS}_{2}$ a été faite par diffraction des rayons $\mathrm{X}$ en utilisant un diffractomètre à source de cobalt dont la raie d'émission $K_{\alpha}$ a une longueur d'onde $\lambda$ égale à $1,79202 \AA$.

Les spectres de transmission $T(\lambda)$ et de réflexion $R(\lambda)$ des couches minces de $\mathrm{CuInS}_{2}$ sont obtenus dans la gamme U.V.-visible et proche infrarouge, à l'aide d'un spectrophotomètre Beckmann U.V. 5240 équipé d'une sphère intégratrice Acta U.V. 520. L'éclairement par réflexion ainsi que par transmission se fait normalement du côté de la couche. Les mesures ont été effectuées à la température ambiante en prenant comme référence, l'air pour la transmission et le sulfate de barium pour la réflexion.

Pour la réalisation et l'étude de la cellule photovoltaïque $\mathrm{CdS} / \mathrm{CuInS}_{2}$, nous avons choisi la configuration Back Wall. Pour cela, nous déposons par la même technique de pulvérisation une couche transparente et conductrice d'oxyde d'étain dopée au fluor $\mathrm{SnO}_{2}$ (F) $\left(0,5 \mu \mathrm{m}, R_{0}=10 \Omega\right)$ sur du verre servant comme contact avant. Cette couche constitue le substrat du semi-conducteur CdS $(4 \mu \mathrm{m})$ que nous formons aussi par la même technique et qui joue le rôle de fenêtre optique dans ce type de cellule [11]. L'hétérojonction étudiée est ensuite formée par la croissance de la couche mince de $\mathrm{CuInS}_{2}$

$$
\left(1 \mu \mathrm{m}, \frac{(\mathrm{Cu}(\mathrm{I}))}{(\mathrm{In}(\mathrm{III}))}=1\right)
$$

sur la bicouche $\mathrm{SnO}_{2}(\mathrm{~F}) / \mathrm{CdS}$. Une couche mince d'or $(700 \AA)$ est déposée en dernière étape par évaporation thermique sous vide $\left(10^{-6}\right.$ torr $)$ constituant ainsi le contact arrière de la photopile.

Les caractéristiques courant tension à l'obscurité et sous éclairement de la cellule sont relevées en utilisant une rampe de tension qui permet une variation continue de la tension de polarisation. La photopile est éclairée du côté $\mathrm{CdS}$ avec une lumière d'intensité $100 \mathrm{~mW} \cdot \mathrm{cm}^{-2}$ ajustée à l'aide d'une cellule étalon S.A.T.

\section{Résultats et discussions.}

3.1 ANAlySE AUX RAYONS X. - La figure 1 représente les diagrammes de diffraction des rayons $\mathrm{X}$ des couches minces de $\mathrm{CuInS}_{2}$ déposées sur verre et sur $\mathrm{SnO}_{2} /$ Verre avec un rapport de concentrations dans la solution à pulvériser $\frac{(\mathrm{Cu}(\mathrm{I}))}{(\mathrm{In}(\mathrm{III}))}$ égal à 0,55 . Ces couches cristallisent suivant la structure chalcopyrite caractérisée par les trois orientations principales (112), (220) et (116).

Nous remarquons la présence d'autres phases secondaires telles que $\operatorname{In}_{2} \mathrm{~S}_{3}$ et $\operatorname{In}_{6} \mathrm{~S}_{7}$. La phase $\mathrm{In}_{2} \mathrm{~S}_{3}$ a été aussi identifiée sur des couches de $\mathrm{CuInS}_{2}$ préparées par évaporation thermique sous vide [13] et par pulvérisation cathodique réactive Sputtering [14]. Les autres pics d'orientations apparus correspondent à la couche de $\mathrm{SnO}_{2}$ dont l'orientation préférentielle (200) est perpendiculaire au substrat.

La faible largeur des différentes raies est typique d'une bonne cristallinité des couches minces de $\mathrm{CuInS}_{2}$. L'orientation préférentielle (112) des cristallites correspondante aux plans parallèles au substrat, est privilégiée quelque soit le substrat utilisé. En effet les valeurs expérimentales des rapports d'intensité $\frac{I_{220}}{I_{112}}$ et $\frac{I_{116}}{I_{112}}$ sont plus petites que les valeurs théoriques correspondant à une répartition aléatoire des cristallites (désordre parfait) $[3,15,16]$. Nous constatons aussi que l'orientation (112) est plus prédominante lorsqu'on passe du substrat verre au substrat $\mathrm{SnO}_{2} /$ Verre (Tab. I). Ces résultats sont proches de ceux obtenus par Grindle et al. [14] sur des couches de $\mathrm{CuInS}_{2}$ préparées par pulvérisation cathodique réactive.

Par ailleurs Chu et al. [17] ont fabriqué des couches minces de $\mathrm{CuInS}_{2}$ par transport en phase vapeur à courte distance Close-spacing-vapor-transport et ont trouvé que les cristallites ont tendance à s'orienter plutôt suivant la direction (220).

Pour un rapport de concentration $\frac{(\mathrm{Cu}(\mathrm{I}))}{(\mathrm{In}(\mathrm{III}))}$ égal à 

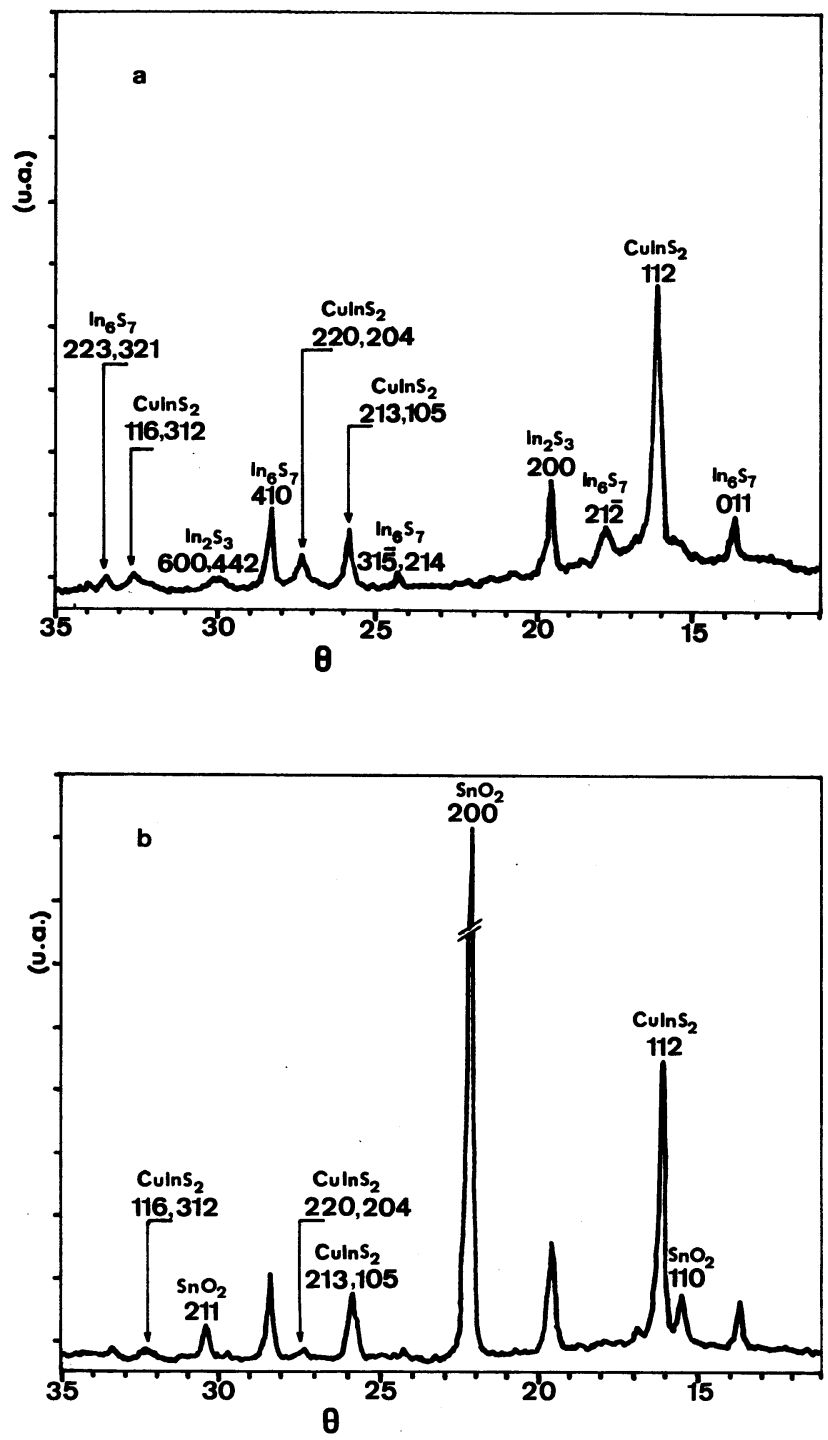

Fig. 1. - Diagramme de diffraction des rayons $\mathrm{X}$ des couches minces de $\mathrm{CuInS}_{2}$ airless spray d'épaisseur $0,3 \mu \mathrm{m}$ $\left(\frac{(\mathrm{Cu}(\mathrm{I}))}{(\mathrm{In}(\mathrm{III}))}=0,55\right) \cdot \mathrm{a}$ - Substrat : verre ; b - Substrat : $\mathrm{SnO}_{2}(\mathrm{~F}) /$ verre.

[X-ray pattern diffraction of $0.3 \mu \mathrm{m}$ thick airless sprayed $\mathrm{CuInS}_{2}$ thin layers $\left(\frac{(\mathrm{Cu}(\mathrm{I}))}{(\operatorname{In}(\mathrm{III}))}=0,55\right)$. a - Substrate : Glass ; b - Substrate : $\mathrm{SnO}_{2}(\mathrm{~F}) /$ Glass.]

1 , nous remarquons que les phases secondaires tendent à disparaître pour une épaisseur de la couche de l'ordre de $1 \mu \mathrm{m}$ (Fig. 2). Dans ce cas, le degré d'orientation (112) est favorisé au détriment de la direction (220). D'autre part une élévation du rapport $\frac{(\mathrm{Cu}(\mathrm{I}))}{(\mathrm{In}(\mathrm{III}))}$ à la valeur 2 ne fait pas apparaître ces phases secondaires et ne perturbe pas la structure des courbes (Fig. 3). Ceci peut être attribué, lorsque la température de substrat est relativement élevée $\left(T_{\mathrm{s}} \geqslant 300^{\circ} \mathrm{C}\right)$, à l'excès d'indium dans la couche plutôt qu'à celui du cuivre.
Tableau I. - Degrés d'orientations (220) et (116) théoriques et expérimentaux $d u \mathrm{CuInS}_{2}(e=0,3 \mu \mathrm{m}$; $\left.T_{\mathrm{s}}=340^{\circ} \mathrm{C} ; \frac{(\mathrm{Cu}(\mathrm{I}))}{(\operatorname{In}(\mathrm{III}))}=0,55\right)$.

[Theoretical and experimental (220) and (116) orientation degrees of $\mathrm{CuInS}_{2}\left(e=0.3 \mu \mathrm{m} ; T_{\mathrm{s}}=340^{\circ} \mathrm{C}\right.$; $\left.\frac{(\mathrm{Cu}(\mathrm{I}))}{(\operatorname{In}(\mathrm{III}))}=0.55\right)$.]

\begin{tabular}{|c|c|c|c|}
\hline \multirow{2}{*}{$\begin{array}{l}\text { Degré } \\
\text { d'orienta- } \\
\text { tion }\end{array}$} & \multirow{2}{*}{ Théorie } & \multicolumn{2}{|c|}{$\begin{array}{c}\text { Expérience } \\
\text { Substrat }\end{array}$} \\
\hline & & Verre & $\mathrm{SnO}_{2}$ \\
\hline$\frac{I_{220}}{I_{112}}$ & 0,8 & 0,104 & 0,033 \\
\hline$\frac{I_{116}}{I_{112}}$ & 0,6 & 0,058 & 0,033 \\
\hline
\end{tabular}
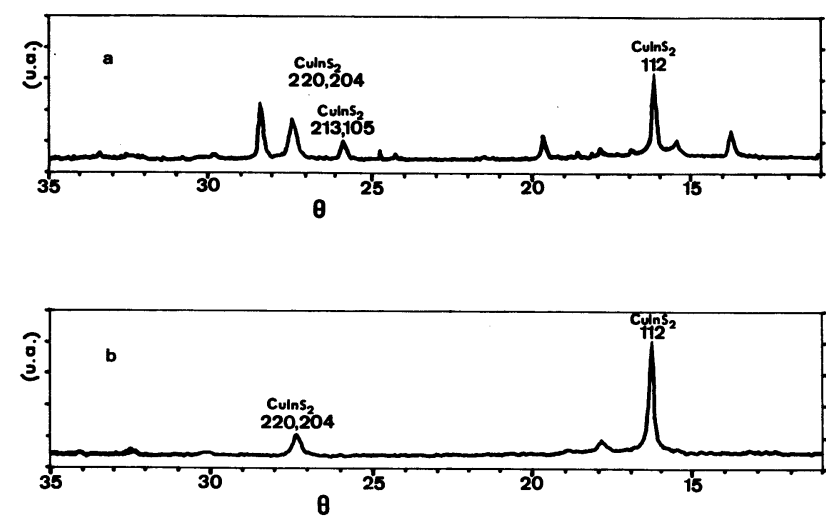

Fig. 2. - Diagramme de diffraction des rayons $\mathbf{X}$ des couches minces de $\mathrm{CuInS}_{2}$ airless spray déposées sur verre $\left(\frac{(\mathrm{Cu}(\mathrm{I}))}{(\operatorname{In}(\mathrm{III}))}=1\right) \cdot \mathrm{a}$ - Epaisseur $e=0,3 \mu \mathrm{m}$; b - Epaisseur $e=1 \mu \mathrm{m}$.

[X-ray pattern diffraction of airless sprayed $\mathrm{CuInS}_{2}$ thin layers deposed on glass $\left(\frac{(\mathrm{Cu}(\mathrm{I}))}{(\operatorname{In}(\mathrm{III}))}=1\right)$. a - Thickness $e=0.3 \mu \mathrm{m} ; \mathrm{b}$ - Thickness $e=1 \mu \mathrm{m}$.]

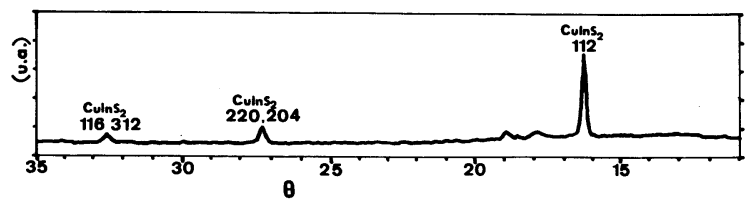

Fig. 3. - Diagramme de diffraction des rayons $\mathrm{X}$ d'une couche mince de $\mathrm{CuInS}_{2}$ airless spray déposée sur verre et d'épaisseur $1 \mu \mathrm{m}\left(\frac{(\mathrm{Cu}(\mathrm{I}))}{(\operatorname{In}(\mathrm{III}))}=2\right)$.

[X-ray pattern diffraction of an $1 \mu \mathrm{m}$ thick airless sprayed $\mathrm{CuInS}_{2}$ thin layer deposed on glass $\left.\left(\frac{(\mathrm{Cu}(\mathrm{I}))}{(\mathrm{In}(\mathrm{III}))}=2\right)\right]$. 
3.2 CARACTÉRISATIONS OPTIQUes. - Sur la figure 4 sont représentés les spectres de transmission $T(\lambda)$ et de réflexion $R(\lambda)$ des couches de $\mathrm{CuInS}_{2}$ déposées sur verre pour deux épaisseurs différentes.

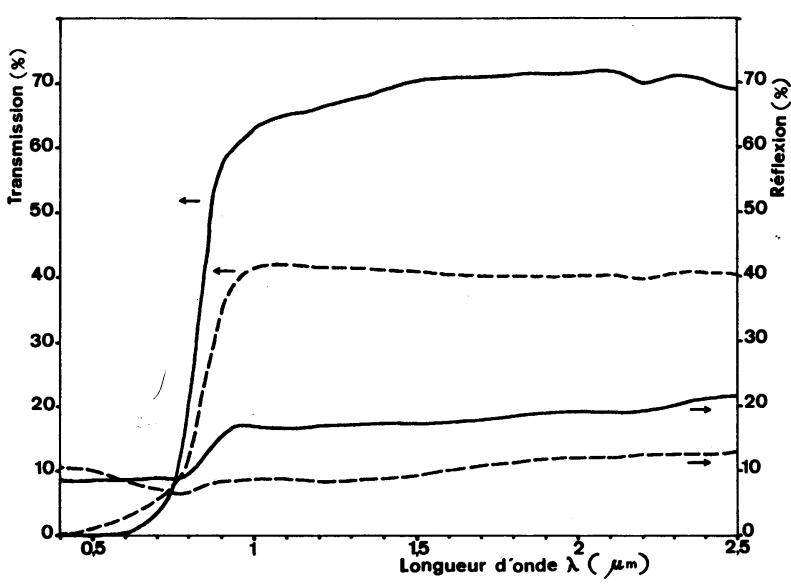

Fig. 4. - Spectres de transmission et de réflexion optiques des couches minces de $\mathrm{CuInS}_{2}$ airless spray déposées sur verre $\left(\frac{(\mathrm{Cu}(\mathrm{I}))}{(\operatorname{In}(\mathrm{III}))}=1\right) \cdot(\longrightarrow)$ Epaisseur $e=$ $0,3 \mu \mathrm{m}$; (----) Epaisseur $e=1 \mu \mathrm{m}$.

[Optical transmission and reflection spectra of airless sprayed $\mathrm{CuInS}_{2}$ thin layers deposed on glass $\left(\frac{(\mathrm{Cu}(\mathrm{I}))}{(\mathrm{In}(\mathrm{III}))}=1\right) \cdot(\longrightarrow)$ Thickness $e=0.3 \mu \mathrm{m}$; (----) Thickness $e=1 \mu \mathrm{m}$.]

Nous observons une très faible transmission des couches dans le visible, région pour laquelle la couche de CdS est transparente [11]. De même, nous constatons que la valeur de transmission $(\simeq 70 \%)$ de la couche d'épaisseur $0,3 \mu \mathrm{m}$, pour des longueurs d'onde supérieures à $1 \mu \mathrm{m}$, est pratiquement la même que celle des couches de CdS préparées par le même procédé de fabrication mais d'épaisseur six fois plus grande $(2 \mu \mathrm{m})$ [11]. Ceci montre que la couche de $\mathrm{CuInS}_{2}$ utilisé est un bon absorbeur. D'autre part, dans la même région $(\lambda>1 \mu \mathrm{m})$, nous remarquons que $T(\lambda)$ et $R(\lambda)$ ne présentent pas de franges d'interférence, ce qui prouve que le pouvoir de diffusion optique de ce type de couche est très important. L'absence de ces franges a été aussi constatée dans le cas des couches de CdS « airless spray » déjà citées. Ceci n'est pas le cas des couches de CdS, ayant la même épaisseur, et formées par évaporation thermique sous vide [18]. La diffusion optique, qui est étroitement liée aux inhomogénéités des couches en surface et en volume (boules de surface, phases secondaires, inclusions, joints de grain, ...) [19], semble ainsi dépendre de la technique de fabrication utilisée.

Le front d'absorption moyenne de ces couches est très net et correspond à des longueurs d'onde comprises entre $0,7 \mu \mathrm{m}$ et $1 \mu \mathrm{m}$. Dans cette zone, l'expression approchée de la transmission est de la forme :

$$
T(\lambda)=(1-R(\lambda))^{2} \exp (-\alpha e)
$$

$e$ est l'épaisseur de la couche et $\alpha$ son coefficient d'absorption. La réflexion à l'interface couche/substrat est supposée négligeable.

L'exploitation des spectres $T(\lambda)$ et $R(\lambda)$ confinés à cette région pour le calcul de l'absorption $\alpha$ en fonction de l'énergie $h \nu$ montre bien que ces couches sont fortement absorbantes $\left(\alpha \geqslant 3 \times 10^{4} \mathrm{~cm}^{-1}\right)$ (Fig. 5).

La valeur de la largeur de bande interdite $E_{g}$ a été déterminée à partir de la relation [20]:

$$
(\alpha h \nu)^{2}=B\left(h \nu-E_{g}\right) \quad(B=\text { constante })
$$

valable dans le cas des transitions directes (Fig. 6).

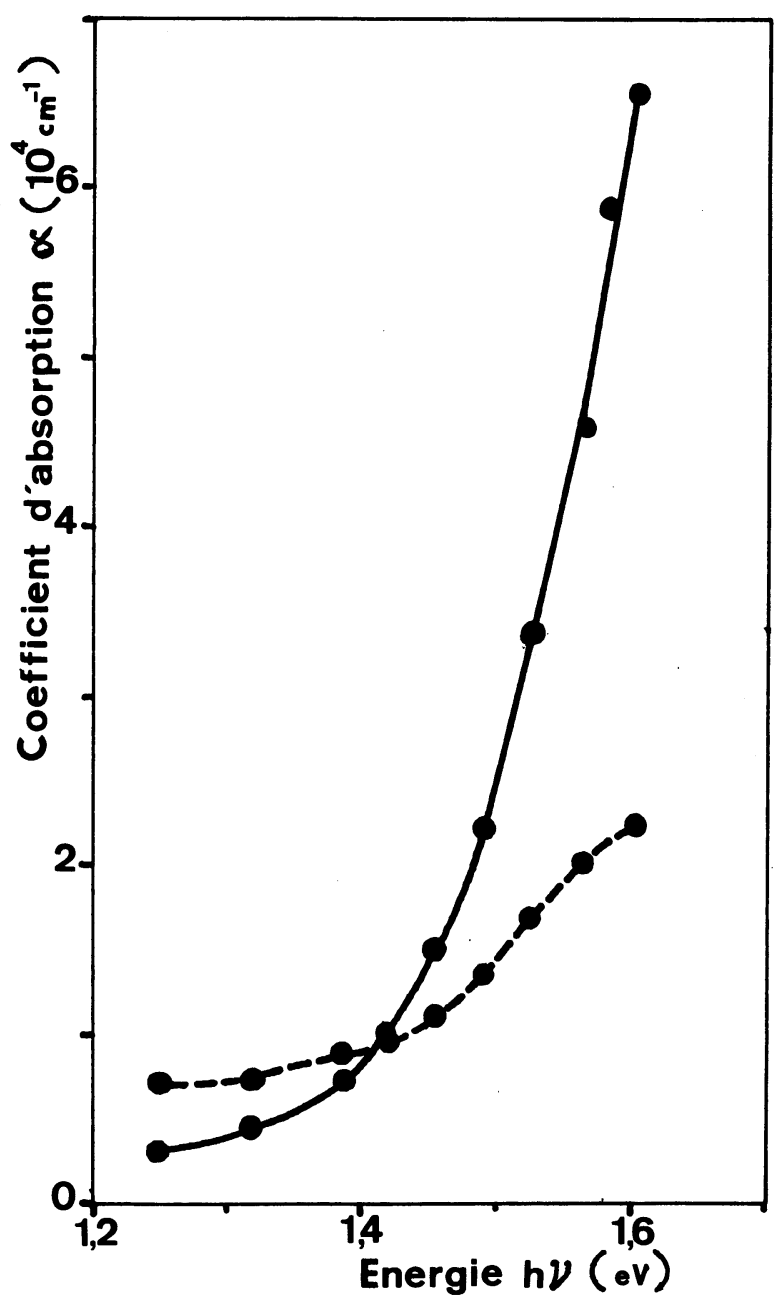

Fig. 5. - Variation du coefficient d'absoprtion $\alpha$ en fonction de l'énergie $h \nu$ des couches minces de $\mathrm{CuInS}_{2}$ airless spray déposées sur verre. ( $\bullet \longrightarrow)$ Epaisseur

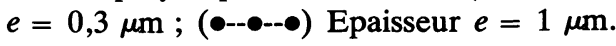

[Variation of the absorption coefficient $\alpha$ as a function of the energy $h \nu$ of airless sprayed $\mathrm{CuInS}_{2}$ thin layers deposed on glass. (๑๐) Thickness $e=0,3 \mu \mathrm{m}$;

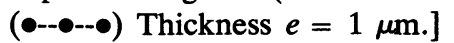




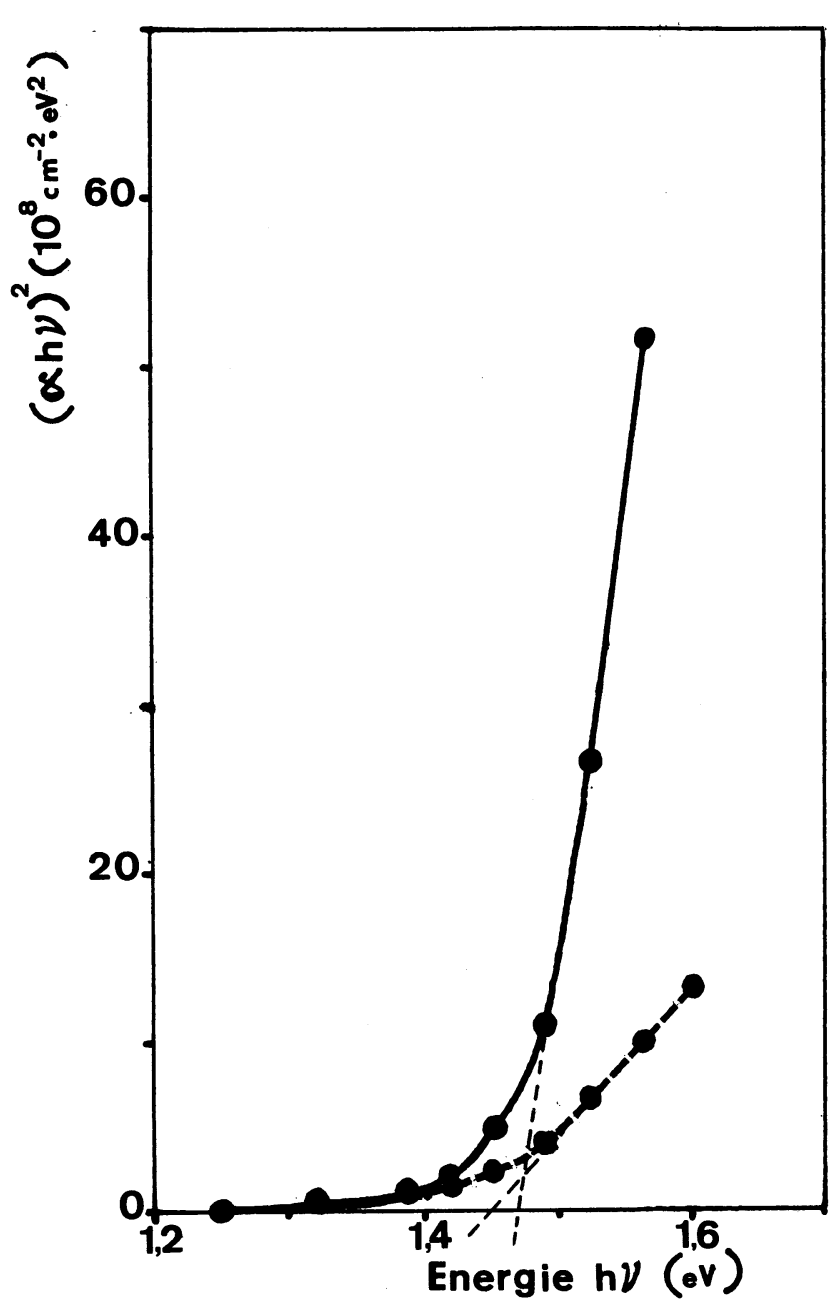

Fig. 6. - Variation de $(\alpha h \nu)^{2}$ en fonction de l'énergie $h \nu$ des couches minces de $\mathrm{CuInS}_{2}$ airless spray déposées

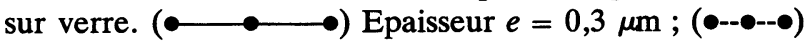
Epaisseur $e=1 \mu \mathrm{m}$.

[Variation of $(\alpha h \nu)^{2}$ as a function of the energy $h \nu$ of airless sprayed $\mathrm{CuInS}_{2}$ thin layers deposed on glass.

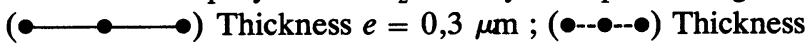
$e=1 \mu \mathrm{m}$.

Les valeurs de $E_{g}$ obtenues pour les épaisseurs $0,3 \mu \mathrm{m}$ et $1 \mu \mathrm{m}$ sont légèrement différentes, soient respectivement $1,47 \mathrm{eV}$ et $1,45 \mathrm{eV}$. Ce léger écart peut être attribué aux inhomogénéités que présentent ces couches. En effet, lors de la déposition un certain nombre de défauts tels que les inclusions et les phases secondaires de caractéristiques différentes de celles du $\mathrm{CuInS}_{2}$ peuvent apparaître dans la couche avec une densité différente d'une épaisseur à une autre et affectent en particulier sa structure (Fig. 2). Le coefficient d'absorption $\alpha(h \nu)$ obtenu correspond alors à un milieu effectif formé par du $\mathrm{CuInS}_{2}$ et de ces perturbations et non du composé seul. Une étude plus rigoureụse tenant compte de ces inhomogénéités doit être reprise pour élaborer une nouvelle approche de calcul qui permette d'expliquer cet écart.
Gorska et al. [4] ont obtenu par des calculs similaires, sur des couches minces de $\mathrm{CuInS}_{2}$ spray, une valeur de $E_{g}$ plus faible, soit $1,42 \mathrm{eV}$. Par ailleurs, Bhattacharya et al. [5] ont effectué des mesures photoélectrochimiques de réponse spectrale (photocourant $J$ en fonction de la longueur d'onde $\lambda$ ) de la jonction $\mathrm{CuInS}_{2}$ (Electrodéposition)/Electrolyte (2 M KOH ; 1,4 M Na $2 \mathrm{~S} ; 2,6 \mathrm{M} \mathrm{S}$ ) sous un éclairement $\mathrm{AM} 1$, et ont déduit, par extrapolation de la courbe $(J h \nu)^{2}$ en fonction de $h \nu$ limitée à la zone d'absorption moyenne, le même ordre de grandeur de $E_{g}(1,45 \mathrm{eV})$. Pour des couches minces de $\mathrm{CuInS}_{2}$ évaporées, Sun et al. [2] ont trouvé plutôt des valeurs de $E_{g}$ relativement élevées de l'ordre de $1,54 \mathrm{eV}$.

Cette dispersion des valeurs de $E_{g}$ provient probablement de l'état cristallin des couches qui sont réalisées par des techniques de fabrication différentes. Dans le cas des couches minces polycristallines, la valeur de $E_{g}$ est en effet étroitement liée au processus de croissance des grains, aux paramètres de réseau et à l'orientation et la stochiométrie des couches [2].

3.3 CARACTÉRISTIQUe COURANT-TENSION DE L'HÉTÉROJONCTION CdS/CuInS ${ }_{2}$. - Sur la figure 7 nous avons représenté les caractéristiques $I-V$ à l'obscurité et sous éclairement de la cellule $\mathrm{CdS} / \mathrm{CuInS}_{2}$ non traitée (surface active $0,125 \mathrm{~cm}^{2}$ ). Nous constatons la manifestation d'un effet photovoltaïque dans l'hétérojonction pour lequel la tension de circuit ouvert $V_{\text {Co }}$ est de $0,3 \mathrm{~V}$ et le courant de court-circuit $I_{\mathrm{cc}}$ est $2,74 \mathrm{~mA} . \mathrm{cm}^{-2}$. Le rendement $\eta$ correspondant est de l'ordre de 0,28\%. En exposant la photopile non encapsulée à l'air et à la température ambiante pendant quarante jours, la tension de circuit ouvert est restée pratiquement inchangée mais le courant de court-circuit a chuté à la valeur $1,98 \mathrm{~mA} . \mathrm{cm}^{-2}$ et s'est stabilisé par la suite. La cellule est devenue ainsi relativement stable.

Dans le tableau II sont rassemblés les paramètres photovoltaïques de la cellule étudiée. La valeur du photocourant $I_{\mathrm{ph}}(3,2 \mathrm{~mA})$ rend bien compte du caractère absorbeur de la couche de $\mathrm{CuInS}_{2}$ dans la cellule. La valeur du facteur de qualité $A(2,2)$ montre que le processus de transport par génération recombinaison est prédominant $[1,6]$. Son accroissement au cours du temps $[3,1]$ peut être expliqué par une contribution de plus en plus importante de l'effet Tunnel [21].

La faible valeur de la résistance shunt $R_{\mathrm{sh}}(186 \Omega)$ montre l'existence d'une fuite importante $\left(I_{\mathrm{s}}=7 \mu \mathrm{A}\right)$ due probablement à la pénétration du $\mathrm{CuInS}_{2}$ formé au cours de la pulvérisation dans les joints de grain de la couche de CdS [11]. Ceci a limité le facteur de forme $F F$ à la valeur $34 \%$.

De même nous observons un grand écart entre les 


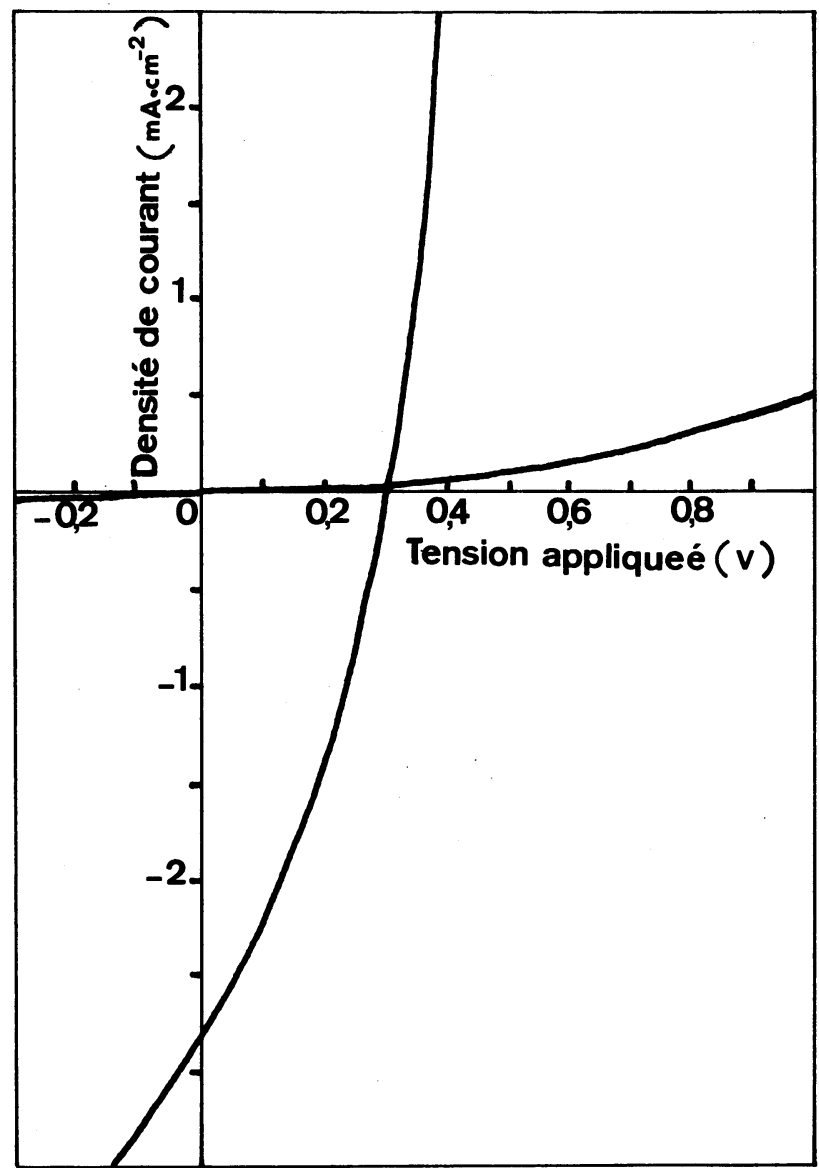

Fig. 7. - Caractéristiques $I-V$ à l'obscurité et sous éclairement de la cellule $\mathrm{SnO}_{2}(\mathrm{~F}) / \mathrm{CdS} / \mathrm{CuInS}_{2} / \mathrm{Au}$.

[ $I-V$ characteristics in darkness and under illumination of $\mathrm{SnO}_{2}(\mathrm{~F}) / \mathrm{CdS} / \mathrm{CuInS} / \mathrm{Au}$ cell.].

valeurs de la résistance série $R_{\mathrm{s}}$ à l'obscurité et sous éclairement ( $2 \mathrm{k} \Omega-31 \mathrm{k} \Omega$ ). Cet écart est apparemment dû au fait que les couches formant la jonction sont très résistantes et qu'en particulier le semiconducteur CdS est photoconducteur.

Les valeurs du facteur de qualité et de la résistance shunt montrent bien qu'un meilleur contrôle de l'interface est nécessaire. En effet la présence du $\mathrm{CuInS}_{2}$ aux joints de grain du CdS réduit énormément les performances photovoltaïques de la cellule. Pour cela, une étude systématique du traitement thermique avant et après l'association des deux matériaux s'avère nécessaire.
De même la nouvelle structure des cellules solaires formées à partir des bicouches de $\mathrm{CuInX} \mathrm{X}_{2}$ telle que $\mathrm{CdS} / \mathrm{CuInSe}_{2}[1,22]$, peut apporter des améliorations à la qualité du type de cellule que nous étudions (Fig. 8).

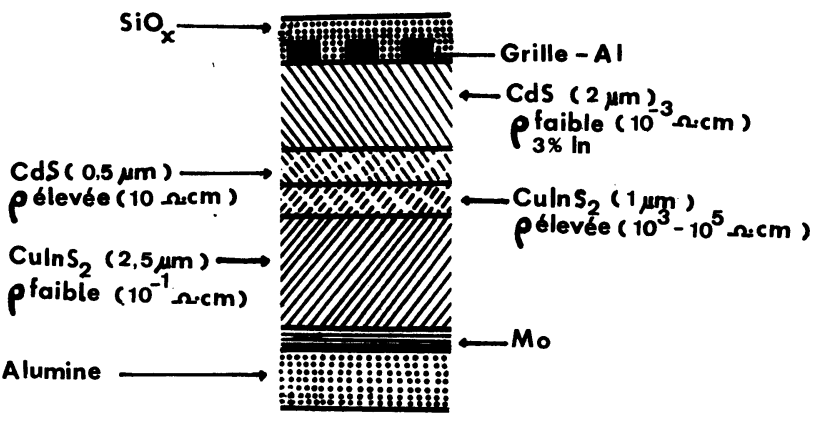

Fig. 8. - Structure de la cellucle $\mathrm{CdS} / \mathrm{CuInSe}_{2}$ réalisée par évaporation thermique sous vide d'après Noufi et al. (22).

[CdS $/ \mathrm{CuInSe}{ }_{2}$ cell structure realized by thermal evaporation in vacuum by Noufi et al. (22)].

Enfin, nous avons reporté dans le tableau III quelques résultats obtenus dans la littérature sur des hétérojonctions similaires réalisées par deux techniques différentes. Kazmerski $[1,6]$ a constaté une importante valeur de $R_{\mathrm{s}}$ qu'il a attribuée en partie au contact arrière $\mathrm{Zn}$-Au de la photopile. Il explique par ailleurs que la réduction de $I_{\mathrm{cc}}$ à des valeurs faibles est due partiellement au fait que l'accord de maille entre le $\mathrm{CdS}$ et le $\mathrm{CuInS}_{2}$ est relativement élevé $(5,56 \%)$. Gorska et al. [4] attribue la très faible valeur de $I_{\text {cc }}$ surtout au fait que la couche de CdS «spray » est fortement résistante $\left(\simeq 200 \Omega \cdot \mathrm{cm}^{-1}\right)$. Ces résultats concordent avec ceux de Wagner (23) obtenus sur des cellules CdS (évaporé)/CuInS 2 (monocristal).

\section{Conclusion.}

Les couches minces de $\mathrm{CuInS}_{2}$ ont été préparées par pulvérisation chimique sans air.

A une température de substrat relativement élevée de l'ordre de $340^{\circ} \mathrm{C}$ et pour des rapports de concen-

Tableau II. - Paramètres photovoltaïques de la cellule $\mathrm{SnO}_{2}(\mathrm{~F}) / \mathrm{CdS} / \mathrm{CuInS} / \mathrm{Au}$.

[Photovoltaic parameters of the $\mathrm{SnO}_{2}(\mathrm{~F}) / \mathrm{CdS} / \mathrm{CuInS}_{2} / \mathrm{Au}$ cell.]

\begin{tabular}{|c|c|c|c|c|c|c|c|c|}
\hline $\begin{array}{c}I_{\mathrm{cc}} \\
\left(\mathrm{mA} \cdot \mathrm{cm}^{-2}\right)\end{array}$ & $\begin{array}{c}V_{\mathrm{co}} \\
(\mathrm{V})\end{array}$ & $F F$ & $\begin{array}{c}R_{\mathrm{s}} \\
(\Omega)\end{array}$ & $\begin{array}{c}R_{\mathrm{sh}} \\
(\Omega)\end{array}$ & $\begin{array}{c}\eta \\
(\%)\end{array}$ & $A$ & $\begin{array}{c}I_{\mathrm{ph}} \\
\left(\mathrm{mA} \cdot \mathrm{cm}^{-2}\right)\end{array}$ & $\begin{array}{c}I_{\mathrm{s}} \\
(\mu \mathrm{A})\end{array}$ \\
\hline 2,74 & 0,3 & 0,34 & 31 & 186 & 0,28 & 2,2 & 3,2 & 7 \\
\hline
\end{tabular}


Tableau III. - Résumé de quelques résultats obtenus sur des hétérojonctions $\mathrm{CdS} / \mathrm{CuInS}_{2}$. [Summary of some results obtained for $\mathrm{CdS} / \mathrm{CuInS}_{2}$ heterojunctions.]

\begin{tabular}{|c|c|c|c|c|c|c|c|}
\hline Conditions de Fabrication & $\begin{array}{l}\text { Traitement } \\
\text { Thermique }\end{array}$ & $\begin{array}{l}\text { Surface } \\
\left(\mathrm{cm}^{2}\right)\end{array}$ & $\begin{array}{l}V_{\mathrm{co}} \\
(\mathrm{V})\end{array}$ & $\begin{array}{c}I_{\mathrm{cc}} \\
\left(\mathrm{mA} \cdot \mathrm{cm}^{-2}\right)\end{array}$ & $F F$ & $\begin{array}{c}\eta \\
(\%)\end{array}$ & Réf. \\
\hline $\begin{array}{l}-\mathrm{CuInS}_{2}(2 \mu \mathrm{m}-4 \mu \mathrm{m}) \text { évaporé } \\
\text { double sources }\left(\mathrm{CuInS}_{2} ; \mathrm{S}\right) \\
T_{\mathrm{s}}=450 \mathrm{~K} \\
-\mathrm{CdS}(4 \mu \mathrm{m}-6 \mu \mathrm{m}) \text { évaporé } \\
T_{\mathrm{s}}=475 \mathrm{~K} \\
\text { Structure Backwall } \\
\text { Contact arrière } \mathrm{Zn}-\mathrm{Au} \\
\text { Contact avant grille }\left(I_{\mathrm{n}}\right)\end{array}$ & $\begin{array}{c}T=355 \mathrm{~K} \\
10 \mathrm{~min} \\
10^{-1} \mathrm{~Pa}\end{array}$ & $\begin{array}{l}0,124 \\
0,25\end{array}$ & $\begin{array}{l}0,47 \\
0,51\end{array}$ & $\begin{array}{l}12,08 \\
12,5\end{array}$ & $\begin{array}{l}0,45 \\
0,51\end{array}$ & $\begin{array}{l}2,55 \\
3,25\end{array}$ & $\begin{array}{l}(1,8) \\
(1,6)\end{array}$ \\
\hline $\begin{array}{l}-\mathrm{CuInS}_{2}(\lesssim 1 \mu \mathrm{m}) \\
\text { Spray } \\
\text { Solution aqueuse : } \mathrm{CuCl}_{2} ; \\
\text { InCl } \mathrm{Cl}_{3} \text { et thiourée }(\text { ou } \mathrm{N}-\mathrm{N} \text {-dimé- } \\
\text { thyl thiourée) acidifiée par } \mathrm{HCl} \text { et } \\
\text { maintenu à } 100^{\circ} \mathrm{C} \\
T_{\mathrm{s}}=180^{\circ} \mathrm{C}-300^{\circ} \mathrm{C} \\
\text { - CdS monocristal }\end{array}$ & $\begin{array}{c}T=400^{\circ} \mathrm{C} \\
15 \text { min } \\
\text { sous vide }\end{array}$ & & 0,1 & 0,04 & & & (4) \\
\hline - CdS spray & & & 0,25 & 0,004 & & & \\
\hline $\begin{array}{l}\text { - } \mathrm{CuInS}_{2} \text { monocristal } \\
\text { - CdS évaporé } T_{\mathrm{s}}=200^{\circ} \mathrm{C}\end{array}$ & & & 0,42 & & & 0,8 & (23) \\
\hline
\end{tabular}

Eclairement sous AM1.

trations de cuivre et d'indium en solution $0,5<\frac{(\mathrm{Cu}(\mathrm{I}))}{(\operatorname{In}(\mathrm{III}))}<2$, les dépôts de $\mathrm{CuInS}_{2}$ obtenus sont bien cristallisés et leur orientation principale (112) est privilégiée.

Nous avons constaté qu'un excès d'indium engendre l'apparition de phases secondaires indésirables tels que $\operatorname{In}_{2} S_{3}$ et $\operatorname{In}_{6} S_{7}$. Pour les couches d'épaisseur $1 \mu \mathrm{m}$ correspondant à un rapport $\frac{(\mathrm{Cu}(\mathrm{I}))}{(\mathrm{In}(\mathrm{III}))}$ égal à 1 , ces phases disparaissent.

$\mathrm{La}$ valeur de la largeur de bande interdite $E_{g}$ obtenue $(1,45 \mathrm{eV}-1,47 \mathrm{eV})$ est proche de l'opti- mum pour la conversion de l'énergie solaire $(1,5 \mathrm{eV})$. Son amélioration nécessite encore une optimisation des conditions expérimentales de fabrication des couches.

L'étude des caractéristiques $I-V$ de la cellule $\mathrm{CdS} / \mathrm{CuInS}_{2}$ et son évolution au cours du temps (sans encapsulation, à l'air et à la température ambiante) ont montré que ce type de photopile est relativement stable. Son étude s'avère donc intéressante et doit être complétée par d'autres caractérisations telles que le contrôle de la stoéchiométrie, de l'état cristallin et des propriétés électriques des couches dans le but de faire des photopiles plus efficaces.

\section{Bibliographie}

[1] Kazmerski, L. L. and Wagner, S., in Current Topics in photovoltaics, CouTts, T. J. and Meakin, J. D. Eds, (Academic Press, New York) 1985, p. 41-109.

[2] Sun, L. Y., Kazmerski, L. L., Clark, A. H., IRELAND, P. J. and MORTON, D. W., J. Vaccum Sci. Technol. 15 (1978) 265.

[3] Shay, J. L. and WeRnick, J. H., Ternary chalcopyrite semiconductors: Growth Electronic Proper- ties and Applications (Pergamon, New York) 1975.

[4] GorsKa, M., BeAulieu, R., Loferski, J. J. and ROESSLER, B., Solar Energy Materials 1 (1979) 313.

[5] Bhattacharya, R. N., Cahen, D. and Hodes, G., Solar Energy Materials 10 (1984) 41.

[6] KAZMerski, L. L., in Ternary Compounds 1977 35, (Inst. Phys., London) 1977, pp. 217-228. 
[7] Kazmerski, L. L. and Sanborn, G. A., J. Appl. Phys. 48 (1977) 3178.

[8] Kazmerski, L. L., White, F. R., Ayyagari, M. S., JuANG, Y. J. and PAterson, R. P., J. Vac. Sci. Technol. 14 (1977) 65.

[9] Levart, M., Thiebaut, B. and Vedel, J., Int. J. Solar Energy 1 (1983) 451.

[10] Dachraoui, M., Belgacem, S., Bennaceur, R. et Latrous, H., J. Soc. Chim. Tunisie 2 (1985) 59.

[11] Ben Said, M. A., Belgacem, S., Dachraoui, M., BENNACEUR, R. et BouchriHA, H., Revue Phys. Appl. 21 (1986) 407.

[12] Chamberlin, R. R. and Skarman, J., J. Electrochem. Soc. 113 (1966) 86.

[13] KaZmerski, L. L., Ayyagari, M. S. and SAnborn, G. A., J. Appl. Phys. 46 (1975) 4865.

[14] Grindle, S.P., Smith, C. W. and Mittleman, S. D., Appl. Phys. Lett. 35 (1979) 24.

[15] Picot and Pierrot, Bull. Soc. fr. Minéral. Cristallogr. 86 (1963) 7.
[16] Kazmerski, L. L., Ayyagari, M. S., Sanborn, G. A., White, F. R. and Merril, A. J., Thin Solid Films 37 (1976) 323.

[17] Chu, T. L., Chu, S. S., Chien, C. P. and Lo, D. H., J. Electrochem. Soc. 132 (1985) 2020.

[18] Diallo, A. O., Thèse de Doctorat de $3^{e}$ cycle, U.S.T.L., Montpellier (1981).

[19] Belgacem, S., Saurel, J. M. and Bougnot, J., Thin Solid Films 92 (1982) 199.

[20] Johnson, E. J., Semiconductors and Semimetals, 3, Willardson, R. K. and BeER, A. C., Eds. (Academic Press, New York) 1967, p. 157.

[21] Milnes, A. G. and FeuCht, D. L., Heterojunctions and Metal-semiconductor Junctions (Academic Press, New York) 1972.

[22] Noufi, R., Souza, P. and Osterwald, C., Solar Cells 15 (1985) 87.

[23] Wagner, S., in Ternary Compounds 1977, vol. 35, HolAH, G. D., Ed. (Inst. Phys., London) 1977, pp. 205-216. 